\title{
On Solitary Wave Solutions for the Camassa-Holm and the Rosenau-RLW-Kawahara Equations with the Dual-Power Law Nonlinearities
}

\author{
Nattakorn Sukantamala ${ }^{1,2}$ and Supawan Nanta $\mathbb{D}^{3}$ \\ ${ }^{1}$ Chiang Mai University, Chiang Mai 50200, Thailand \\ ${ }^{2}$ Advanced Research Center for Computational Simulation, Chiang Mai University, Chiang Mai 50200, Thailand \\ ${ }^{3}$ Phetchabun Rajabhat University, Phetchabun 67000, Thailand
}

Correspondence should be addressed to Supawan Nanta; nanta.supawan@gmail.com

Received 2 January 2021; Revised 10 June 2021; Accepted 5 July 2021; Published 19 July 2021

Academic Editor: Sheng Zhang

Copyright (c) 2021 Nattakorn Sukantamala and Supawan Nanta. This is an open access article distributed under the Creative Commons Attribution License, which permits unrestricted use, distribution, and reproduction in any medium, provided the original work is properly cited.

\begin{abstract}
The nonlinear wave equation is a significant concern to describe wave behavior and structures. Various mathematical models related to the wave phenomenon have been introduced and extensively being studied due to the complexity of wave behaviors. In the present work, a mathematical model to obtain the solution of the nonlinear wave by coupling the classical Camassa-Holm equation and the Rosenau-RLW-Kawahara equation with the dual term of nonlinearities is proposed. The solution properties are analytically derived. The new model still satisfies the fundamental energy conservative property as the original models. We then apply the energy method to prove the well-posedness of the model under the solitary wave hypothesis. Some categories of exact solitary wave solutions of the model are described by using the Ansatz method. In addition, we found that the dual term of nonlinearity is essential to obtain the class of analytic solution. Besides, we provide some graphical representations to illustrate the behavior of the traveling wave solutions.
\end{abstract}

\section{Introduction}

In the study of nonlinear wave phenomena, the nonlinear partial differential equations are one of the great mathematical models to investigate the problems. A variety of the mathematical theory for the wave equations has been achieved theoretically and numerically, arising in empirical applications on ion-acoustic and magnetohydrodynamics waves in plasma, longitudinal dispersive waves in elastic rods, pressure waves in liquid-gas bubble mixtures, and rotating flow down a tube. For instance, the various phenomena of shallow-water waves are led by nonlinear partial differential equations such as the Korteweg-de Vries (KdV) equation [1-7], the Benjamin-Bona-Mahony (BBM) equation [8-11], the Symmetric Regularized Long Wave (SRLW) equation [12-15], the Kawahara equation [16-19], and the Rosenau equation [20-23]. For further understanding of nonlinear behaviors of shallow-water waves, the generalized Rosenau-RLW equation was introduced in the following:

$$
u_{t}-u_{x x t}+u_{x x x x t}+u_{x}+\beta u^{p} u_{x}=0
$$

where $p \geq 1$ and $\beta$ are a constant. Equation (1) is an extension of the Rosenau equation by adding a viscous term $-u_{x x t}$ and replacing the nonlinear term with a general power of nonlinearity $u^{p} u_{x}$. If $p=1$ and $\beta=1$, then equation (1) is called usual Rosenau-RLW equation. When $p=2$, then equation (1) is called the modified Rosenau-RLW equation. For numerical study for the Rosenau-RLW equation, we refer to [24-27]. Later, many models related to the Rosenau and the Rosenau-RLW equations have been studied and become an essential topic in the study of shallow-water wave behavior. In [28], the solitons and periodic solutions for the Rosenau-KdV and Rosenau-Kawahara equations were 
obtained utilizing the sine-cosine and the tanh methods. Wongsaijai and Poochinapan [29] numerically studied the Rosenau-RLW-KdV equation by coupling the RosenauRLW equation and the Rosenau-KdV equation. In [30], Labidi and Biswas obtained the analytical one-soliton solution for the Rosenau-Kawahara equation by using He's semi-inverse variational principle. Biswas et al. [31] obtained the solitary solution and two invariance of the generalized Rosenau-Kawahara equation with power law nonlinearity. Recently, by adding a viscous term $-u_{x x t}$ into the RosenauKawahara equation, which is called the Rosenau-RLWKawahara equation,

$$
u_{t}-u_{x x t}+u_{x x x x t}+u_{x}+u_{x x x}-u_{x x x x x}+\beta u^{p} u_{x}=0 .
$$

It has been a growing interest in computation nonlinear wave equations. In [32], $\mathrm{He}$ and Pan initially studied a second-order three-level linearly implicit difference scheme which is energy-conserved and unconditionally stable. In [33], two conservative high-order accurate finite difference schemes for the periodic initial value generalized RosenauKawahara-RLW equation were introduced and extensively studied. For more related nonlinear wave equations, readers can refer to [34-43].

As furthermore consideration of the unidirectional shallow-water waves, one of the equations is a CamassaHolm $(\mathrm{CH})$ equation which can be founded:

$$
u_{t}+\kappa u_{x}-u_{x x t}+3 u u_{x}=2 u_{x} u_{x x}+u u_{x x x}
$$

where $\kappa$ is a constant. The equation has been derived by Camassa and Holm [44] in 1993 and has a solitary peaked solution which discontinuity in the first derivative. For the significance of $\kappa$, it was shown in [45] that for all $\kappa>0$, there are smooth solitary wave solutions, and for $\kappa=0$, it has peaked soliton solution (peakon). A classification of weak traveling wave solution of Camassa-Holm equation was given in [46]. Furthermore, Kalisch and Lenells have investigated the kind of traveling wave solution, smooth traveling waves, cusped traveling waves, and composited traveling waves [47]. The orbital stability of the peakons and the solitons of the smooth solitary wave of $\mathrm{CH}$ equation were shown in [46, 48, 49]. In 2010, Lai [50] established the existence and uniqueness of a local solution of the $\mathrm{CH}$ equation in Sobolev space $H^{s}(\mathbb{R})$, and the well-posedness was established by $\mathrm{Li}$ and Olver [49]. Very recently, Nanta et al. [51] obtained the numerical study of the generalized Camassa-Holm equation involving dual-power law nonlinearities. Other studies of $\mathrm{CH}$-related equation are also reported by various publications [52-57].

In this paper, our purpose is to investigate the coupling of the original $\mathrm{CH}$ equation and the Rosenau-RLW-Kawahara equation with the dual-power law nonlinearity:

$$
\begin{aligned}
u_{t} & -\mu u_{x x t}+2 \kappa u_{x}+\eta u_{x x x}+u_{x x x x t}+\gamma u_{x x x x x} \\
& =f(u) u_{x}+s\left[2 u_{x} u_{x x}+u u_{x x x}\right]
\end{aligned}
$$

with the initial condition

$$
u(x, 0)=u_{0}(x), x \in\left[x_{L}, x_{R}\right]
$$

where $u_{0}(x)$ is a known smooth function, $\kappa, \eta \in \mathbb{R}, \mu>0$. The function $f(u)=A u+B u^{m}$ represents the dispersive nonlinear terms in both low and high-order nonlinearity, where $A$, $B \in \mathbb{R}$, and $m \in \mathbb{N}$ indicates the power law nonlinearity. Moreover, the solitary wave solution and its derivatives have the following asymptotic values:

$$
u \longrightarrow 0 \text { as } x \longrightarrow \pm \infty \text {, and for } n \geq 1, \frac{\partial^{n} u}{\partial x^{n}} \longrightarrow 0 \text { as } x \longrightarrow \pm \infty
$$

Note that equation (4) reduces to Rosenau-RLWKawahara equation when $s=A=0$, and it reduces to $\mathrm{CH}$ equation (3) when $\mu=1, \kappa=1 / 2, \eta=0, \gamma=0, B=0$, and $s=1$ and removing viscous term $u_{x x x x t}$. Moreover, when $A=\gamma=\eta$ $=s=0$, equation (4) reduces to the Rosenau-RLW equation.

To study the nature of solutions, researchers have attempted to find the exact solution of the Rosenau-type equation. Many methods were introduced and developed to explore the analytical solution corresponding to nonlinear partial differential equations. By using the sech and trigonometric function method, Esfahani [58] (Esfahani and Pourgholi [59]) studied solitary wave solutions to the generalized Rosenau-KdV and Rosenau-RLW equation, respectively. The solitons and shock waves were discussed by Razborave et al. [60] by applying a semi-inverse variational method. In [29], Wongsaijai and Poochinapan used the sine-cosine method to find the exact solution of the Rosenau-RLW-KdV equation. He and Pan [32] also used the sine-cosine method to obtain the solitary solution for the generalized Rosenau-Kawahara-RLW equation, and the solution for the Rosenau-Kawahara-RLW equation with, notably, the generalized Novikov type perturbation was solely derived by $\mathrm{He}$ [38]. The solution of $(2+1)$ dimensional of nonlinear wave equation using modified exponential function method and Ansatz function technique with symbolic computation was proposed in [61]. In [62], solitary wave solution for Ablowitz-Kaup-NewellSegur water wave equation was obtained by using the simple equation method and modified simple equation method. The generalized extended tanh method and the Fexpansion method were used to derive exact solutions for the Kadomtsev-Petviashvili and the modified KadomtsevPetviashvili dynamical equations [63]. In addition, readers can refer to $[64,65]$ for more methods to find analytic wave solutions.

The paper has been organized as follows. In Section 2, the fundamental energy-preserving property of the initial boundary value problems is proved. By applying the energy method, the well-posedness of the new model is obtained in the solution space $H_{0}^{2}(\Omega)$. In addition, the traveling wave solutions of the equation were employed by the Ansatz method, which determines solitary solutions and periodic 
solutions. Finally, concluding remarks are reported in the last section.

\section{Solution Properties}

We first state that the solution of equations (4)-(6) satisfies the following energy conservative property.

Theorem 1. If the solution of equations (4)-(6) $u$ and its derivatives $\partial_{x} u, \partial_{x}^{2} u$ go to zero when $|x| \longrightarrow \infty$, then equations (4)-(6) have the following global conservation law:

$$
\begin{aligned}
E(t) & =\int_{-\infty}^{\infty} u^{2}(x, t)+\mu u_{x}^{2}(x, t)+u_{x x}^{2}(x, t) d x \\
& =\int_{-\infty}^{\infty} u^{2}(x, 0)+\mu u_{x}^{2}(x, 0)+u_{x x}^{2}(x, 0) d x=E(0),
\end{aligned}
$$

for all $t \in[0, T]$.

Proof. Let $u_{t}-\mu u_{x x t}+u_{x x x x t}=-2 \kappa u_{x}-\eta u_{x x x}+\gamma u_{x x x x x}+f(u)$ $u_{x}+s\left(2 u_{x} u_{x x}+u u_{x x x}\right)$. Then,

$$
\begin{aligned}
\frac{d E(t)}{d t}= & 2 \int_{-\infty}^{\infty} u u_{t} d x+2 \mu \int_{-\infty}^{\infty} u_{x} u_{x t} d x+2 \int_{-\infty}^{\infty} u_{x x} u_{x x t} d x \\
= & 2 \int_{-\infty}^{\infty} u u_{t} d x-2 \mu \int_{-\infty}^{\infty} u u_{x x t} d x+2 \int_{-\infty}^{\infty} u u_{x x x x t} d x \\
= & 2 \int_{-\infty}^{\infty} u\left(-2 \kappa u_{x}-\eta u_{x x x}-\gamma u_{x x x x x}+f(u) u_{x}\right. \\
& \left.+s\left(2 u_{x} u_{x x}+u u_{x x x}\right)\right) d x .
\end{aligned}
$$

Using the integration by parts and the assumption $u$ and its derivatives $\partial_{x} u, \partial_{x}^{2} u \longrightarrow 0$ as $|x| \longrightarrow \infty$, we obtain

$$
\begin{aligned}
\int_{-\infty}^{\infty} u u_{x} d x & =0, \\
\int_{-\infty}^{\infty} u u_{x x x} d x & =0, \\
\int_{-\infty}^{\infty} u u_{x x x x x} d x & =0, \\
\int_{-\infty}^{\infty} u f(u) u_{x} d x & =\int_{-\infty}^{\infty}\left(A u^{2}+B u^{m+1}\right) d u \\
& =\lim _{x_{L} \longrightarrow-\infty x_{R} \longrightarrow \infty}\left[\left.\frac{A}{3} u^{3}\right|_{x_{L}}+\left.\frac{B}{m+2} u^{m+2}\right|_{x_{L}} ^{x_{R}}\right]=0 \\
\int_{-\infty}^{\infty} u\left(2 u_{x} u_{x x}+\right. & \left.u u_{x x x}\right) d x=\int_{-\infty}^{\infty} u\left(u_{x} u_{x x}+\left(u u_{x x}\right)_{x}\right) d x \\
& =\int_{-\infty}^{\infty} u u_{x} u_{x x} d x-\int_{-\infty}^{\infty} u u_{x} u_{x x} d x=0 .
\end{aligned}
$$

Therefore, $E(t)$ is a constant function, that is,

$$
\frac{d}{d t} \int_{-\infty}^{\infty} u^{2}(x, t)+\mu u_{x}^{2}(x, t)+u_{x x}^{2}(x, t) d x=0
$$

which yields $E(t)=E(0)$ for all $t \in[0, T]$, as desired.

By assumption (6), problem (4) can be set up in a compact subset of $\mathbb{R}$, namely, $\Omega=\left[x_{L}, x_{R}\right]$. Thereby, we consider the following initial-boundary value problem (4) with the initial condition (5) and the boundary conditions

$$
\begin{aligned}
u\left(x_{L}, t\right) & =u\left(x_{R}, t\right)=0 u_{x}\left(x_{L}, t\right)=u_{x}\left(x_{R}, t\right)=0 \text { and } u_{x x}\left(x_{L}, t\right) \\
& =u_{x x}\left(x_{R}, t\right)=0 t, \in[0, T] .
\end{aligned}
$$

For a nonnegative integer $k$, let $H^{k}(\Omega)$ denote the usual Sobolev space of real valued functions defined on the interval $\Omega$. We define the following Sobolev space:

$$
H_{0}^{k}(\Omega)=\left\{u \in H^{k}(\Omega) \mid \frac{\partial^{i} u}{\partial x^{i}}=0 \text { on } \partial \Omega, i=1,2, \cdots, k-1\right\} .
$$

The solutions of equations (4) and (5) with the boundary condition (11) satisfy the following energy conservative property.

Theorem 2. Suppose $u_{0} \in H_{0}^{2}(\Omega)$; then, the solution of equations (4), (5), and (11) satisfies the following:

$$
\begin{aligned}
E(t) & =\int_{x_{L}}^{x_{R}} u^{2}(x, t)+\mu u_{x}^{2}(x, t)+u_{x x}^{2}(x, t) d x \\
& =\int_{x_{L}}^{x_{R}} u^{2}(x, 0)+\mu u_{x}^{2}(x, 0)+u_{x x}^{2}(x, 0) d x=E(0),
\end{aligned}
$$

for all $t \in[0, T]$.

It should be pointed out that the invariant function $E(t)$ indicates the energy conservation for equations (4) and (5). Next, we provide the well-posedness of problems (4) and (5) with the boundary condition (11) on the solution space $H_{0}^{2}\left[x_{L}, x_{R}\right]$. Before providing the well-posedness, we first state the existence, which can be proved by the standard energy method. By combining the local existence and uniqueness with Theorem 2, we obtain the global existence. Therefore, we leave the proof.

Lemma 3 (existence). Suppose $u_{0} \in H_{0}^{2}\left[x_{L}, x_{R}\right]$; then, there exists a positive constant $\delta$ such that $\|u\|_{H^{2}} \leq \delta$, and then, the initial value of problems (4) and (5) has a unique global solution $u(x, t)$ with $u(x, t) \in C\left(0, \infty ; H^{2}\left[x_{L}, x_{R}\right]\right)$.

Theorem 4. Suppose $u_{0} \in H_{0}^{2}\left[x_{L}, x_{R}\right]$; then, problems (4) and (5) with the boundary condition (11) are well-posed. 
Proof. First, we let $u_{1}$ and $u_{2}$ are two solutions of (4) and (5) with the boundary condition (11) satisfying the initial conditions $u_{0,1}$ and $u_{0,2}$, respectively. Let $\varepsilon=u_{1}-u_{2}$; then, by substituting, $\delta$ corresponds to the following equation:

$$
\begin{aligned}
\varepsilon_{t}- & \mu \varepsilon_{x x t}+2 \kappa \varepsilon_{x}+\eta \varepsilon_{x x x}+\varepsilon_{x x x x t}+\gamma \varepsilon_{x x x x x} \\
= & f\left(u_{1}\right)\left(u_{1}\right)_{x}-f\left(u_{2}\right)\left(u_{2}\right)_{x}+s\left[2\left(u_{1}\right)_{x}\left(u_{1}\right)_{x x}+\left(u_{1}\right)\left(u_{1}\right)_{x x x}\right] \\
& \quad-s\left[2\left(u_{2}\right)_{x}\left(u_{2}\right)_{x x}+\left(u_{2}\right)\left(u_{2}\right)_{x x x}\right] .
\end{aligned}
$$

with the initial conditions

$$
\varepsilon(x, 0)=u_{0,1}-u_{0,2}
$$

and boundary conditions

$$
\begin{aligned}
\varepsilon\left(x_{L}, t\right) & =\varepsilon\left(x_{R}, t\right)=0 \varepsilon_{x}\left(x_{L}, t\right)=\varepsilon_{x}\left(x_{R}, t\right) \\
& =0 \text { and } \varepsilon_{x x}\left(x_{L}, t\right)=\varepsilon_{x x}\left(x_{R}, t\right)=0 t, \in[0, T],
\end{aligned}
$$

where $t \in[0, T]$ and $x \in\left[x_{L}, x_{R}\right]$. By the standard energy method, we introduce the following energy function

$$
E^{*}(t)=\int_{x_{L}}^{x_{R}} \varepsilon^{2}+\mu \varepsilon_{x}^{2}+\varepsilon_{x x}^{2} d x
$$

By similar arguments as that in the proof of Theorem 1, we have

$$
\begin{aligned}
\frac{d E^{*}(t)}{d t}= & 2 \int_{x_{L}}^{x_{R}} \varepsilon \varepsilon_{t} d x+2 \mu \int_{x_{L}}^{x_{R}} \varepsilon_{x} \varepsilon_{x t} d x+2 \int_{x_{L}}^{x_{R}} \varepsilon_{x x} \varepsilon_{x x t} d x \\
= & 2 \int_{x_{L}}^{x_{R}} \varepsilon \varepsilon_{t} d x-2 \mu \int_{x_{L}}^{x_{R}} \varepsilon \varepsilon_{x x t} d x+2 \int_{x_{L}}^{x_{R}} \varepsilon \varepsilon_{x x x x t} d x \\
= & 2 \int_{x_{L}}^{x_{R}} \varepsilon\left(-2 \kappa \varepsilon_{x}-\eta \varepsilon_{x x x}-\gamma \varepsilon_{x x x x x}\right) d x \\
& +2 \int_{x_{L}}^{x_{R}} \varepsilon\left[f\left(u_{1}\right)\left(u_{1}\right)_{x}-f\left(u_{2}\right)\left(u_{2}\right)_{x}\right] d x \\
& +2 s \int_{x_{L}}^{x_{R}} \varepsilon\left[2\left(u_{1}\right)_{x}\left(u_{1}\right)_{x x}+\left(u_{1}\right)\left(u_{1}\right)_{x x x}\right. \\
& \left.-\left(2\left(u_{2}\right)_{x}\left(u_{2}\right)_{x x}+\left(u_{2}\right)\left(u_{2}\right)_{x x x}\right)\right] d x \\
= & 2 \int_{x_{L}}^{x_{R}} \varepsilon\left[f\left(u_{1}\right)\left(u_{1}\right)_{x}-f\left(u_{2}\right)\left(u_{2}\right)_{x}\right] d x \\
& +2 s \int_{x_{L}}^{x_{R}} \varepsilon\left[2\left(\left(u_{1}\right)_{x}\left(u_{1}\right)_{x x}-\left(u_{2}\right)_{x}\left(u_{2}\right)_{x x}\right)\right. \\
& \left.+\left(\left(u_{1}\right)\left(u_{1}\right)_{x x x}-\left(u_{2}\right)\left(u_{2}\right)_{x x x}\right)\right] d x .
\end{aligned}
$$

Noting that the first nonlinear term can be estimated as

$$
\begin{aligned}
\int_{x_{L}}^{x_{R}} \varepsilon\left[f\left(u_{1}\right)\left(u_{1}\right)_{x}-f\left(u_{2}\right)\left(u_{2}\right)_{x}\right] \\
=A \int_{x_{L}}^{x_{R}} \varepsilon\left(\varepsilon\left(u_{1}\right)_{x}+u_{2} \varepsilon_{x}\right) d x+2 s B \int_{x_{L}}^{x_{R}} \varepsilon \\
\quad \times\left(\varepsilon_{x}\left(u_{2}\right)^{m}+\varepsilon\left(u_{1}\right)_{x} \sum_{k=1}^{m-1}\left(u_{1}\right)^{m-k-1}\left(u_{2}\right)^{k}\right) \\
\leq C \int_{x_{L}}^{x_{R}}\left|\varepsilon^{2}+\varepsilon \varepsilon_{x}\right| d x \leq C\left(\int_{x_{L}}^{x_{R}} \varepsilon^{2} d x+\int_{x_{L}}^{x_{R}} \varepsilon_{x}^{2} d x\right),
\end{aligned}
$$

where Theorem 1 and the Cauchy-Schwarz inequality are used. For the second term, we see that

$$
\begin{aligned}
2 s \int_{x_{L}}^{x_{R}} \varepsilon & {\left[2\left(\left(u_{1}\right)_{x}\left(u_{1}\right)_{x x}-\left(u_{2}\right)_{x}\left(u_{2}\right)_{x x}\right)\right.} \\
& \left.+\left(\left(u_{1}\right)\left(u_{1}\right)_{x x x}-\left(u_{2}\right)\left(u_{2}\right)_{x x x}\right)\right] d x \\
= & 4 s \int_{x_{L}}^{x_{R}} \varepsilon\left(\left(u_{1}\right)_{x}\left(u_{1}\right)_{x x}-\left(u_{2}\right)_{x}\left(u_{2}\right)_{x x}\right) d x \\
& +2 s \int_{x_{L}}^{x_{R}} \varepsilon\left(\left(u_{1}\right)\left(u_{1}\right)_{x x x}-\left(u_{2}\right)\left(u_{2}\right)_{x x x}\right) d x:=M_{1}+M_{2} .
\end{aligned}
$$

For the term $M_{1}$, by Theorem 1 and the Cauchy-Schwarz inequality, we have

$$
M_{1}=4 s \int_{x_{L}}^{x_{R}} \varepsilon\left(\varepsilon_{x}\left(u_{1}\right)_{x x}+\left(u_{2}\right)_{x} \varepsilon_{x x}\right) d x \leq C \int_{x_{L}}^{x_{R}} \varepsilon^{2} d+\varepsilon_{x}^{2}+\varepsilon_{x x}^{2} d x
$$
$M_{2}$ as

Next, by simple calculations, we can estimate the term

$$
\begin{aligned}
M_{2}= & 2 s \int_{x_{L}}^{x_{R}} \varepsilon\left(\left(u_{1}\right)\left(u_{1}\right)_{x x x}-\left(u_{2}\right)\left(u_{2}\right)_{x x x}\right) d x \\
= & -2 s \int_{x_{L}}^{x_{R}}\left(\left(\varepsilon u_{1}\right)_{x}\left(u_{1}\right)_{x x}-\left(\varepsilon u_{2}\right)_{x}\left(u_{2}\right)_{x x}\right) d x \\
= & -2 s \int_{x_{L}}^{x_{R}} \varepsilon\left(\left(u_{1}\right)_{x}\left(u_{1}\right)_{x x}-\left(u_{2}\right)_{x}\left(u_{2}\right)_{x x}\right) \\
& +\varepsilon_{x}\left(u_{1}\left(u_{1}\right)_{x x}-u_{2}\left(u_{2}\right)_{x x}\right) d x \\
= & -2 s \int_{x_{L}}^{x_{R}} \varepsilon\left(\varepsilon_{x}\left(u_{1}\right)_{x x}+\left(u_{2}\right)_{x} \varepsilon_{x x}\right)+\varepsilon_{x}\left(\varepsilon\left(u_{1}\right)_{x x}+u_{2} \varepsilon_{x x}\right) d x \\
\leq & 2 s \int_{x_{L}}^{x_{R}}\|\varepsilon\|_{\infty}\left|\varepsilon_{x}\right|\left|\left(u_{1}\right)_{x x}\right|+C\left(|\varepsilon|\left|\varepsilon_{x}\right|+\left|\varepsilon_{x}\right|\left|\varepsilon_{x x}\right|\right) d x \\
\leq & C \int_{x_{L}}^{x_{R}} \varepsilon^{2}+\varepsilon_{x}^{2}+\varepsilon_{x x}^{2} d x+s\|\varepsilon\|_{\infty}^{2} \int_{x_{L}}^{x_{R}}\left(u_{1}\right)_{x x}^{2} d x \\
\leq & C \int_{x_{L}}^{x_{R}} \varepsilon^{2}+\varepsilon_{x}^{2}+\varepsilon_{x x}^{2} d x,
\end{aligned}
$$




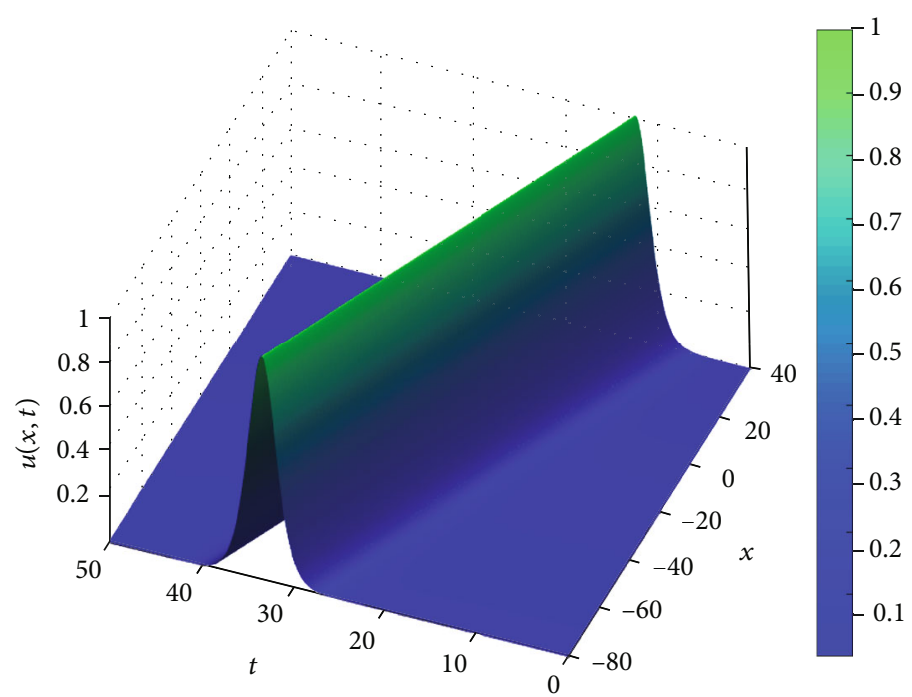

(a) $m=2$

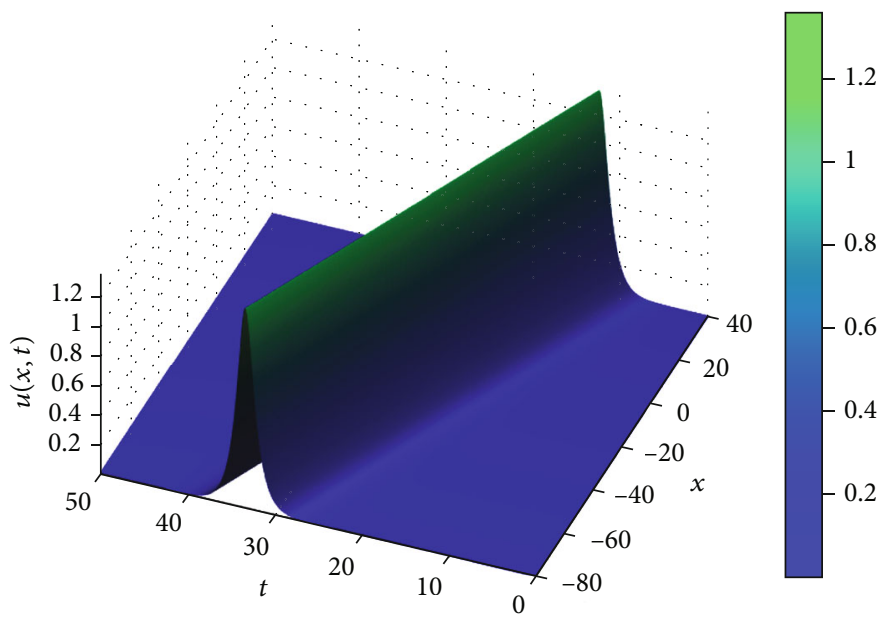

(b) $m=4$

Figure 1: Plot solitary wave solutions (32) when $A=-1$ and $s=2$.

where Theorem 1, the Cauchy-Schwarz inequality, and the Sobolev's inequality are used. Substituting equations (19)-(22) into equation (18) gives

$$
\frac{d E^{*}(t)}{d t} \leq C E^{*}(t)
$$

which yields $E^{*}(t) \leq e^{C T} E^{*}(0)$ for all $t \in[0, T]$. Obviously, the uniqueness is consequently obtained when the initial conditions for $u_{1}$ and $u_{2}$ are the same. Moreover, if $\varepsilon(x, 0)<\delta, \varepsilon_{x}$ $(x, 0)<\delta$, and $\varepsilon_{x x}(x, 0)<\delta$, then we have

$$
\frac{d E^{*}(t)}{d t} \leq e^{C T} E^{*}(0) \leq \delta e^{C T}
$$

for all $t \in[0, T]$. That is, the solution is continuously dependent on the initial condition. Since the existence and uniqueness are obtained by Lemma 3, therefore equations (4) and (5) with the boundary condition (11) are well-posed as required.

\section{Solitary Wave Solutions}

Next, we focus on problems (4) and (5). By introducing $\xi=$ $x-c t$, we see that equation (4) reduces to

$$
\begin{aligned}
& -c u_{\xi}+\mu c u_{\xi \xi \xi}+2 \kappa u_{\xi}+\eta u_{\xi \xi \xi}-c u_{\xi \xi \xi \xi \xi}+\gamma u_{\xi \xi \xi \xi \xi} \\
& =f(u) u_{\xi}+s\left[2 u_{\xi} u_{\xi \xi}+u u_{\xi \xi \xi}\right],
\end{aligned}
$$

that is,

$$
\begin{aligned}
& {[2 \kappa-c] u_{\xi}+[\mu c+\eta] u_{\xi \xi \xi}+[\gamma-c] u_{\xi \xi \xi \xi \xi}} \\
& =f(u) u_{\xi}+s\left[2 u_{\xi} u_{\xi \xi}+u u_{\xi \xi \xi}\right]
\end{aligned}
$$

where $f(u)=A u+B u^{m}$. The solitary wave Ansatz method admits the used assumption

$$
u(\xi)=\lambda \sec ^{\beta}(\alpha \xi)
$$




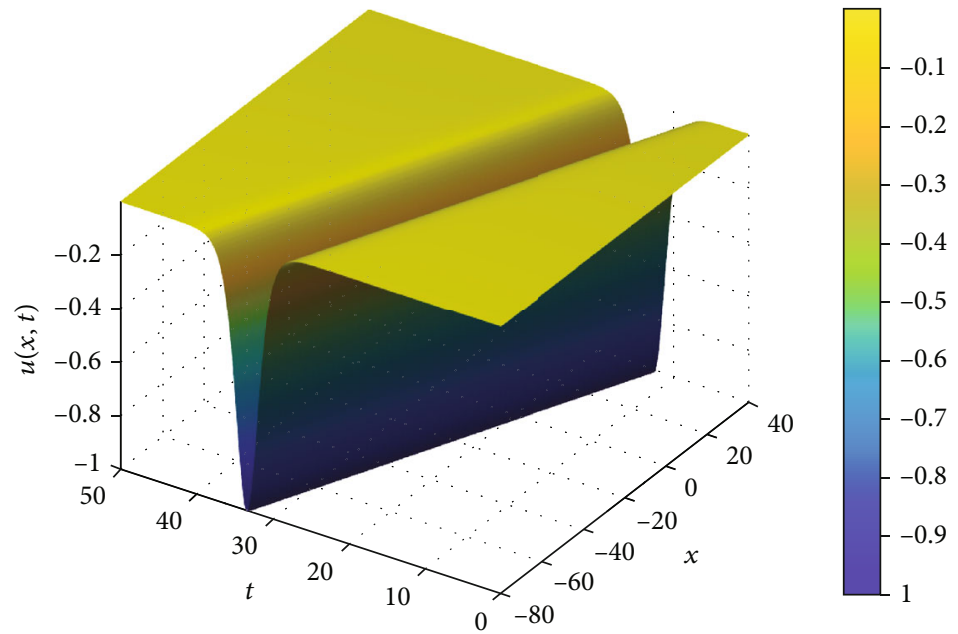

(a) $m=2$

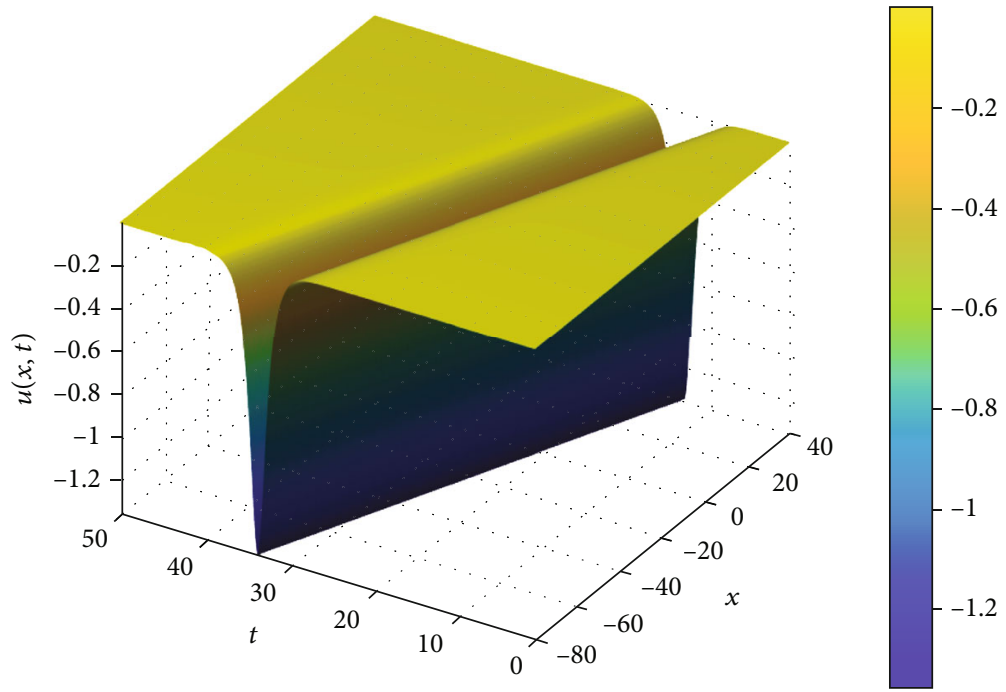

(b) $m=4$

Figure 2: Plot solitary wave solutions (32) when $A=1$ and $s=-2$.

Simple calculations give

$$
\begin{aligned}
u_{\xi}= & -\lambda \alpha \beta \operatorname{sech}^{\beta}(\alpha \xi) \tanh (\alpha \xi), \\
u_{\xi \xi}= & \lambda \alpha^{2} \beta^{2} \operatorname{sech}^{\beta}(\alpha \xi)-\lambda \alpha^{2} \beta(\beta+1) \operatorname{sech}^{\beta+2}(\alpha \xi), \\
u_{\xi \xi \xi}= & \lambda \alpha^{3} \beta(\beta+1)(\beta+2) \operatorname{sech}^{\beta+2}(\alpha \xi) \tanh (\alpha \xi) \\
& -\lambda \alpha^{3} \beta^{3} \operatorname{sech}^{\beta}(\alpha \xi) \tanh (\alpha \xi), \\
u_{\xi \xi \xi \xi}= & \lambda \alpha^{4} \beta^{4} \operatorname{sech}^{\beta}(\alpha \xi)-\lambda \alpha^{4} \beta(\beta+1) \\
& \cdot\left(2 \beta^{2}+4 \beta+4\right) \operatorname{sech}^{\beta+2}(\alpha \xi)+\lambda \alpha^{4} \beta(\beta+1)(\beta+2) \\
& \cdot(\beta+3) \operatorname{sech}^{\beta+4}(\alpha \xi), \\
u_{\xi \xi \xi \xi \xi}= & -\lambda \alpha^{5} \beta^{5} \operatorname{sech}^{\beta}(\alpha \xi) \tanh (\alpha \xi)+\lambda \alpha^{5} \beta(\beta+1)(\beta+2) \\
& \cdot\left(2 \beta^{2}+4 \beta+4\right) \operatorname{sech}^{\beta+2}(\alpha \xi) \tanh (\alpha \xi) \\
& -\lambda \alpha^{5} \beta(\beta+1)(\beta+2)(\beta+3)(\beta+4) \operatorname{sech}^{\beta+4} \\
& \cdot(\alpha \xi) \tanh (\alpha \xi) .
\end{aligned}
$$

Therefore, equation (26) turns into

$$
\begin{aligned}
{[-(2 \kappa} & \left.+c)-(\mu c+\eta) \alpha^{2} \beta^{2}-(\gamma-c) \alpha^{4} \beta^{4}\right] \lambda \alpha \beta \operatorname{sech}^{\beta}(\alpha \xi) \\
& +\left[(\mu c+\eta)+(\gamma-c)\left(2 \beta^{2}+4 \beta+4\right) \alpha^{3}\right] \lambda \alpha^{3} \beta(\beta+1) \\
& \times(\beta+2) \operatorname{sech}^{\beta+2}(\alpha \xi)-(\gamma-c) \lambda \alpha^{5} \beta(\beta+1)(\beta+2) \\
& \times(\beta+3)(\beta+4) \operatorname{sech}^{\beta+4}(\alpha \xi) \\
= & \left.-A \lambda^{2} \beta-3 s \lambda^{2} \alpha^{3} \beta^{3}\right] \operatorname{sech}^{2 \beta}(\alpha \xi)+s \lambda^{2} \alpha^{3} \beta(\beta+1) \\
& \times(3 \beta+2) \operatorname{sech}^{2 \beta+2}(\alpha \xi)-B \lambda^{m+1} \alpha \beta \sec ^{(m+1) \beta}(\alpha \xi) .
\end{aligned}
$$

Balancing sec $\mathrm{h}^{2 \beta+2}(\alpha \xi)$ and $\sec ^{(m+1) \beta}(\alpha \xi)$, we obtain that $2 \beta+2=(m+1) \beta$; so, $\beta=1 / m-1$.

Setting the coefficients of each term of $\sec ^{j}(\mu \xi)$ to zero, we have the following system:

$$
-A \alpha \lambda^{2} \beta-3 s \lambda^{2} \alpha^{3} \beta^{3}=0,
$$




$$
\begin{array}{r}
s \lambda^{2} \alpha^{3} \beta(\beta+1)(3 \beta+2)-B \lambda^{m+1} \alpha \beta=0 \\
(2 \kappa+c)+(\mu c+\eta) \alpha^{2} \beta^{2}+(\gamma-c) \alpha^{4} \beta^{4}=0 \\
(\mu c+\eta)+(\gamma-c)\left(2 \beta^{2}+4 \beta+4\right) \alpha^{3}=0 \\
(\gamma-c) \lambda \alpha^{5} \beta(\beta+1)(\beta+2)(\beta+3)(\beta+4)=0 .
\end{array}
$$

Solving system (30), we obtain the set of parameters.

$$
\begin{aligned}
& \alpha=\left[\frac{-A(m-1)^{2}}{12 s}\right]^{1 / 2}, \\
& \lambda=\left[\frac{-A\left(m^{2}+3 m+2\right)}{6 B}\right]^{1 /(m-1)}, \\
& \gamma=c=-\frac{\eta}{\mu} \\
& \kappa=-\frac{\eta}{2 \mu} .
\end{aligned}
$$

For $s A<0$, we can obtain the following solitary wave solutions for equation (4):

$$
\begin{aligned}
u(x, t)= & {\left[\frac{-A\left(m^{2}+3 m+2\right)}{6 B}\right]^{1 / m-1} \sec h^{2 / m-1} } \\
& \cdot\left((m-1) \sqrt{\frac{-A}{12 s}}\left(x+\frac{\eta}{\mu} t\right)\right) .
\end{aligned}
$$

Additionally, the following periodic wave solutions for equation (4) can be obtained when $s A>0$

$$
\begin{aligned}
u(x, t)= & {\left[\frac{-A\left(m^{2}+3 m+2\right)}{6 B}\right]^{1 / m-1} \sec ^{2 / m-1} } \\
& \cdot\left((m-1) \sqrt{\frac{A}{12 s}}\left(x+\frac{\eta}{\mu} t\right)\right) .
\end{aligned}
$$

Figures 1 and 2 plot the analytical solutions in the case of $A=-1$ and $s=2$ and $A=1$ and $s=-2$, respectively, when $m=2$ and $m=4$.

\section{Concluding Remarks}

In this paper, we successfully studied the nonlinear wave equation by coupling the classical Camassa-Holm equation and the Rosenau-RLW-Kawahara equation in the case of asymptotic boundary conditions. Based on the boundary conditions, we obtained that the equation possesses the conservative energy, which was used to derive the well-posedness in $H_{0}^{2}(\Omega)$. Moreover, to seek the analytic solution in $H_{0}^{2}(\Omega)$, we applied the Ansatz method to derive the solitary wave solution class by balancing linear and nonlinear terms. One can see that the dual term of nonlinearity $f(u)=A u+B u^{m}$ is essential to derive the class of analytic solutions.
In view of Theorem 4, the order of the highest-order derivative appearing in equation (4) is five, but there are six boundary conditions as defined in equation (11), which seems that it is overdetermined for the problem on a bounded interval. It should be pointed out that the boundary condition (11) is logical to study under the solitary wave conditions, that is, $u$ and its derivative approach to zero when $|x| \longrightarrow \infty$ (see equation (6)). However, there are many qualitative differences in the behavior of solutions depending on the number of boundary conditions used. Therefore, this question should be of interest in the future.

\section{Data Availability}

No data were available in the manuscript.

\section{Conflicts of Interest}

No conflict of interest exists. We wish to confirm that there are no known conflicts of interest associated with this publication, and there has been no significant financial support for this work that could have influenced its outcome.

\section{Authors' Contributions}

All authors developed the theoretical formalism and performed the analytic calculations to the writing final version of the manuscript.

\section{Acknowledgments}

This research was supported by Chiang Mai University.

\section{References}

[1] D. J. Korteweg and G. de Vries, "XLI.On the change of form of long waves advancing in a rectangular canal, and on a new type of long stationary waves," Philosophical Magazine, vol. 39, no. 240, pp. 422-443, 1895.

[2] S. Ozer and S. Kutluay, "An analytical-numerical method for solving the Korteweg-de Vries equation," Applied Mathematics and Computation, vol. 164, no. 3, pp. 789-797, 2005.

[3] A. R. Bahadir, "Exponential finite-difference method applied to Korteweg-de Vries equation for small times," Applied Mathematics and Computation, vol. 160, no. 3, pp. 675-682, 2005.

[4] K. Poochinapan, B. Wongsaijai, and T. Disyadej, "Efficiency of high-order accurate difference schemes for the Korteweg-de Vries equation," Mathematical Problems in Engineering, vol. 2014, Article ID 862403, 8 pages, 2014.

[5] X. Lv, T. Shao, and J. Chen, "The study of the solution to a generalized KdV-mKdV equation," Abstract and Applied Analysis, vol. 2013, Article ID 249043, 17 pages, 2013.

[6] A. R. Seadawy, "Two-dimensional interaction of a shear flow with a free surface in a stratified fluid and its solitary-wave solutions via mathematical methods," The European Physical Journal Plus, vol. 518, p. 11, 2017.

[7] M. Elbadri, S. A. Ahmed, Y. T. Abdalla, and W. Hdidi, "A new solution of time-fractional coupled $\mathrm{KdV}$ equation by using natural decomposition method," Abstract and Applied Analysis, vol. 2020, Article ID 3950816, 9 pages, 2020. 
[8] J. L. Bona, W. G. Pritchard, and L. R. Scott, "Numerical schemes for a model for nonlinear dispersive waves," Journal of Computational Physics, vol. 60, no. 2, pp. 167-186, 1985.

[9] D. Bhardwaj and R. Shankar, "A computational method for regularized long wave equation," Computers \& Mathematcs with Applications, vol. 40, no. 12, pp. 1397-1404, 2000.

[10] A. Dogan, "Numerical solution of regularized long wave equation using Petrov-Galerkin method," Communications in Numerical Methods in Engineering, vol. 17, no. 7, pp. 485494, 2001

[11] A. Dogan, "Numerical solution of RLW equation using linear finite elements within Galerkin's method," Applied Mathematical Modelling, vol. 26, no. 7, pp. 771-783, 2002.

[12] C. E. Seyler and D. L. Fenstermacher, "A symmetric regularized-long-wave equation," Physics of Fluids, vol. 27, no. 1, pp. 4-7, 1984.

[13] S. Yimnet, B. Wongsaijai, T. Rojsiraphisal, and K. Poochinapan, "Numerical implementation for solving the symmetric regularized long wave equation," Applied Mathematics and Computation, vol. 273, pp. 809-825, 2016.

[14] S. Li, "Numerical study of a conservative weighted compact difference scheme for the symmetric regularized long wave equations," Numerical Methods for Partial Differential Equations, vol. 35, no. 1, pp. 60-83, 2019.

[15] J. Kerdboon, S. Yimnet, B. Wongsaijai, T. Mouktonglang, and K. Poochinapan, "Convergence analysis of the higher-order global mass-preserving numerical method for the symmetric regularized long-wave equation," International Journal of Computer Mathematics, vol. 98, no. 5, pp. 869-902, 2021.

[16] A. Biswas, "Solitary wave solution for the generalized Kawahara equation," Applied Mathematics Letters, vol. 22, no. 2, pp. 208-210, 2009.

[17] R. Chousurin, T. Mouktonglang, B. Wongsaijai, and K. Poochinapan, "Performance of compact and non-compact structure preserving algorithms to traveling wave solutions modeled by the Kawahara equation," Numerical Algorithms, vol. 85, no. 2, pp. 523-541, 2020.

[18] G.-W. Wang and T.-Z. Xu, "Group analysis and new explicit solutions of simplified modified Kawahara equation with variable coefficients," Abstract and Applied Analysis, vol. 2013, Article ID 139160, 8 pages, 2013.

[19] A. Atangana, N. Bildik, and S. C. O. Noutchie, "New iteration methods for time-fractional modified nonlinear Kawahara equation," Abstract and Applied Analysis, vol. 2014, Article ID 740248, 9 pages, 2014.

[20] P. Rosenau, "A quasi-continuous description of a nonlinear transmission line," Physica Scripta, vol. 34, no. 6B, pp. 827829, 1986

[21] P. Rosenau, "Dynamics of dense discrete systems," Progress in Theoretical Physics, vol. 79, no. 5, pp. 1028-1042, 1988.

[22] M. A. Park, "On the Rosenau equation," Computation and Applied Mathematics, vol. 9, pp. 145-152, 1990.

[23] M. A. Park, "Pointwise decay estimate of solutions of the generalized Rosenau equation," Journal of the Korean Mathematical Society, vol. 29, pp. 261-280, 1992.

[24] X. Pan and L. Zhang, "On the convergence of a conservative numerical scheme for the usual Rosenau-RLW equation," Applied Mathematical Modelling, vol. 36, no. 8, pp. 3371$3378,2012$.

[25] X. Pan and L. Zhang, "Numerical simulation for general Rosenau-RLW equation: an average linearized conservative scheme," Mathematical Problems in Engineering, vol. 2012, Article ID 517818, 15 pages, 2012.

[26] B. Wongsaijai, K. Poochinapan, and T. Disyadej, “A compact finite difference method for solving the general RosenauRLW equation," IAENG International Journal of Applied Mathematics, vol. 44, pp. 192-199, 2014.

[27] B. Wongsaijai, T. Mouktonglang, N. Sukantamala, and K. Poochinapan, "Compact structure-preserving approach to solitary wave in shallow water modeled by the Rosenau-RLW equation," Applied Mathematics and Computation, vol. 340, pp. 84-100, 2019.

[28] J.-M. Zuo, "Solitons and periodic solutions for the Rosenau$\mathrm{KdV}$ and Rosenau-Kawahara equations," Applied Mathematics and Computation, vol. 215, no. 2, pp. 835-840, 2009.

[29] B. Wongsaijai and K. Poochinapan, "A three-level average implicit finite difference scheme to solve equation obtained by coupling the Rosenau-KdV equation and the RosenauRLW equation," Applied Mathematics and Computation, vol. 245, pp. 289-304, 2014.

[30] M. Labidi and A. Biswas, "Application of He's principles to Rosenau-Kawahara equation," Mathematics in Engineering, Science \& Aerospace (MESA), vol. 2, pp. 183-197, 2011.

[31] A. Biswas, H. Triki, and M. Labidi, "Bright and dark solitons of the Rosenau-Kawahara equation with power law nonlinearity," Physics of Wave Phenomenon, vol. 19, no. 1, pp. 24-29, 2011.

[32] D. He and K. Pan, "A linearly implicit conservative difference scheme for the generalized Rosenau-Kawahara-RLW equation," Applied Mathematics and Computation, vol. 271, pp. 323-336, 2015

[33] A. Ghiloufi, M. Rahmeni, and K. Omrani, "Convergence of two conservative high-order accurate difference schemes for the generalized Rosenau-Kawahara-RLW equation," Engineering with Computers, vol. 36, no. 2, pp. 617-632, 2020.

[34] M. Mei, "Long-time behavior of solution for Rosenau-Burgers equation (I)," Applicable Analysis, vol. 63, no. 3-4, pp. 315330, 1996.

[35] M. Mei, "Long-time behaviour of solution for RosenauBurgers equation(II)," Applicable Analysis, vol. 68, no. 3-4, pp. 333-356, 1998.

[36] M. Mei, "Large-time behavior of solution for generalized Benjamin-Bona-Mahony-Burgers equations," Nonlinear Analysis, vol. 33, no. 7, pp. 699-714, 1998.

[37] L. Liu and M. Mei, "A better asymptotic profile of RosenauBurgers equation," Applied Mathematics and Computation, vol. 131, no. 1, pp. 147-170, 2002.

[38] D. He, "Exact solitary solution and a three-level linearly implicit conservative finite difference method for the generalized Rosenau-Kawahara-RLW equation with generalized Novikov type perturbation," Nonlinear Dynamics, vol. 85, no. 1, pp. 479-498, 2016.

[39] N. Tamang, B. Wongsaijai, T. Mouktonglang, and K. Poochinapan, "Novel algorithm based on modification of Galerkin finite element method to general Rosenau-RLW equation in (2+1)-dimensions," Applied Numerical Mathematics, vol. 148, pp. 109-130, 2020.

[40] J. Janwised, B. Wongsaijai, T. Mouktonglang, and K. Poochinapan, "A modified three-level average linearimplicit finite difference method for the Rosenau-Burgers equation," Advances in Mathematical Physics, vol. 2014, Article ID 734067, 11 pages, 2014 
[41] S. Ozer, "Numerical solution by quintic B-spline collocation finite element method of generalized Rosenau-Kawahara equation," The Mathematical Scientist, 2021.

[42] B. Wongsaijai, P. Charoensawan, T. Chaobankoh, and K. Poochinapan, "Advance in compact structure-preserving manner to the Rosenau-Kawahara model of shallow-water wave," Mathematical Methods in the Applied Sciences, vol. 44, no. 8, pp. 7048-7064, 2021.

[43] B. Wongsaijai and K. Poochinapan, "Optimal decay rates of the dissipative shallow water waves modeled by coupling the Rosenau-RLW equation and the Rosenau-Burgers equation with power of nonlinearity," Applied Mathematics and Computation, vol. 405, article 126202, 2021.

[44] R. Camassa and D. D. Holm, "An integrable shallow water equation with peaked solitons," Physical Review Letters, vol. 71, no. 11, pp. 1661-1664, 1993.

[45] R. Camassa, D. D. Holm, and J. M. Hyman, "A new integrable shallow water equation," Advances in Applied Mechanics, vol. 31, pp. 1-33, 1994.

[46] J. Lenells, "Traveling wave solutions of the Camassa-Holm equation," Journal of Differential Equations, vol. 217, no. 2, pp. 393-430, 2005.

[47] H. Kalisch and J. Lenells, "Numerical study of traveling-wave solutions for the Camassa-Holm equation," Chaos, Solitons and Fractals, vol. 25, no. 2, pp. 287-298, 2005.

[48] A. Constantin and W. A. Strauss, "Stability of the CamassaHolm solitons," Journal of Nonlinear Science, vol. 12, no. 4, pp. 415-422, 2002.

[49] Y. A. Li and P. J. Olver, "Well-posedness and blow-up solutions for an integrable nonlinearly dispersive model wave equation," Journal of Differential Equations, vol. 162, no. 1, pp. 27-63, 2000.

[50] S. Lai, "The existence and uniqueness of the local solution for a Camassa-Holm type equation," Applied Mathematics and Computation, vol. 216, no. 4, pp. 1287-1298, 2010.

[51] S. Nanta, S. Yimnet, K. Poochinapan, and B. Wongsaijai, “On the identification of nonlinear terms in the generalized Camassa-Holm equation involving dual-power law nonlinearities," Applied Numerical Mathematics, vol. 160, pp. 386-421, 2021.

[52] K. H. Kwek, H. Gao, W. Zhang, and C. Qu, "An initial boundary value problem of Camassa-Holm equation," Journal of Mathematical Physics, vol. 41, no. 12, pp. 8279-8285, 2000.

[53] A. Bressan and A. Constantin, "Global conservative solutions of the Camassa-Holm equation," Archive for Rational Mechanics and Analysis, vol. 183, no. 2, pp. 215-239, 2007.

[54] J. Escher and Z. Yin, "Initial boundary value problems of the Camassa-Holm equation," Communications in Partial Differential Equations, vol. 33, no. 3, pp. 377-395, 2008.

[55] H. Bulut, T. A. Sulaiman, F. Erdogan, and H. M. Baskonus, "On the new hyperbolic and trigonometric structures to the simplified MCH and SRLW equations," The European Physical Journal Plus, vol. 132, p. 12, 2017.

[56] X. Zong, X. Cheng, Z. Wang, and Z. Han, "Initial boundary value problem and asymptotic stabilization of the twocomponent Camassa-Holm equation," Abstract and Applied Analysis, vol. 2011, Article ID 635851, 20 pages, 2011.

[57] Z. Yin, "Several dynamic properties of solutions to a generalized Camassa-Holm equation," Abstract and Applied Analysis, vol. 2013, Article ID 247841, 5 pages, 2013.
[58] A. Esfahani, "Solitary wave solutions for generalized RosenauKdV equation," Communications in Theoretical Physics, vol. 55, no. 3, pp. 396-398, 2011.

[59] A. Esfahani and R. Pourgholi, "Dynamics of solitary waves of the Rosenau-RLW equation," Differential Equations and Dynamical Systems, vol. 22, no. 1, pp. 93-111, 2014.

[60] P. Razborova, B. Ahmed, and A. Biswas, "Solitons, shock waves and conservation laws of Rosenau-KdV-RLW equation with power law nonlinearity," Applied Mathematics \& Information Sciences, vol. 8, no. 2, pp. 485-491, 2014.

[61] H. M. Baskonus, "Complex surfaces to the fractional $(2+1)$ dimensional Boussinesq dynamical model with the local Mderivative," The European Physical Journal Plus, vol. 134, p. 10, 2019.

[62] A. Ali, A. R. Seadawy, and D. Lu, "Computational methods and traveling wave solutions for the fourth-order nonlinear Ablowitz-Kaup-Newell-Segur water wave dynamical equation via two methods and its applications," Open Physics, vol. 16, pp. 219-226, 2018.

[63] A. R. Seadawy and K. El-Rashidy, "Dispersive solitary wave solutions of Kadomtsev-Petviashvili and modified KadomtsevPetviashvili dynamical equations in unmagnetized," Results in Physics, vol. 8, pp. 1216-1222, 2018.

[64] A. R. Seadawy, "Ion acoustic solitary wave solutions of twodimensional nonlinear Kadomtsev-Petviashvili-Burgers equation in quantum plasma," Mathematical Methods in the Applied Sciences, vol. 40, no. 5, pp. 1598-1607, 2017.

[65] M. Arshad, A. R. Seadawy, and D. Lu, "Elliptic function and solitary wave solutions of the higher-order nonlinear Schrödinger dynamical equation with fourth-order dispersion and cubic-quintic nonlinearity and its stability," The European Physical Journal Plus, vol. 371, p. 11, 2017. 